\title{
Modelado Matemático de Zombis, una presentación divertida de modelos epidemiológicos (reseña)
}

Mathematical Modelling of

Zombies, a fun presentation of epidemiological models (review)

José Camilo Herrera Hurtado

DOI: $10.29151 /$ reit.n1a4 


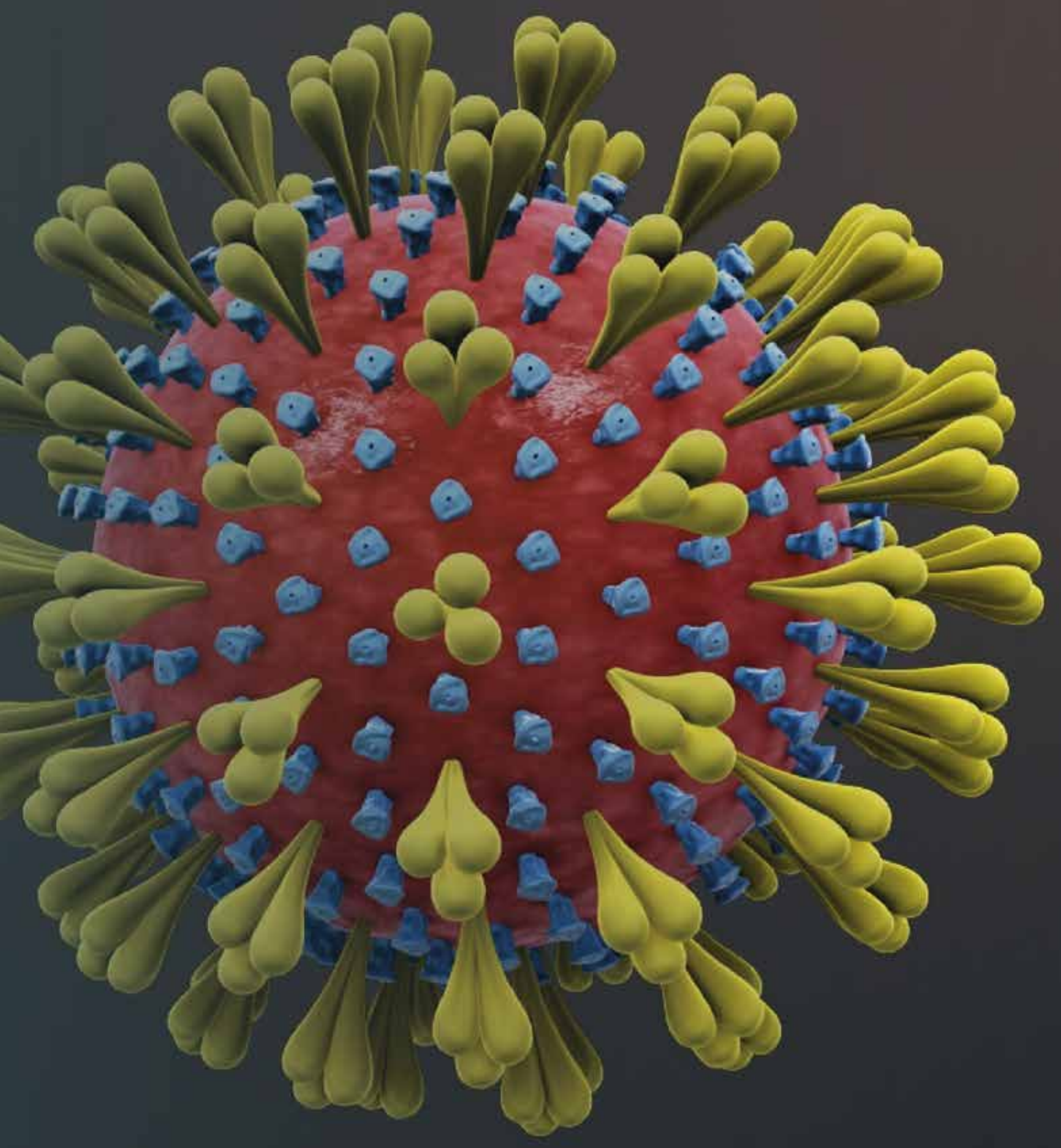




\section{Resumen}

Se presenta el libro editado por Robert Smith en 2014, Ilamado "Mathematical Modelling of Zombies", el cual realiza un análisis epidemiológico comparando el efecto de zombis y vampiros como agentes propagadores de enfermedad. Con base en ese caso hipotético, presenta una introducción a la inferencia estadística, al modelamiento basado en agente, enjambres de organismos biológicos, técnicas de difusión, modelos lineales evolutivos, toma de decisiones por lógica difusa, métodos de regresión estadística, y redes sociales; entre otros modelos matemáticos.

Para esta reseña nos enfocaremos en el capítulo "The Undead: A Plague on Humanity or a Powerful New Tool for Epidemiological Research" escrito por Jane M. Heffernan y Derek J. Wilson. Este capítulo trata con dos situaciones diferentes: enfermedades epidémicas y enfermedades endémicas, cuya diferencia consiste en que la primera se refiere a una condición ocasional, mientras que la otra consiste en una condición permanente con la que toca convivir. Se tratará con el modelo de propagación de enfermedades SIR, cuyas ecuaciones diferenciales se resolverán con ayuda del programa libre GNU Octave.

Palabras clave: Ecuaciones diferenciales; SIR; análisis epidemiológico; GNU Octave. 


\begin{abstract}
The book edited by Robert Smith in 2014, called "Mathematical Modeling of Zombies", is presented, which performs an epidemiological analysis comparing the effect of zombies and vampires as disease spreading agents. Based on that hypothetical case; presents an introduction to statistical inference, agent-based modeling, swarms of biological organisms, diffusion techniques, evolutionary linear models, fuzzy logic decision-making, statistical regression methods, and social media; among other mathematical models.
\end{abstract}

For this review we will focus on the chapter "The Undead: A Plague on Humanity or a Powerful New Tool for Epidemiological Research" written by Jane M. Heffernan and Derek J. Wilson. This chapter deals with two different situations: epidemic diseases and endemic diseases, the difference being that the first refers to an occasional condition, while the other consists of a permanent condition with which it is time to live. It will be treated with the SIR disease propagation model, whose differential equations will be solved with the help of the free software GNU Octave.

Keywords: Differential equations; SIR; epidemiological analysis; GNU Octave. 


\section{Introducción}

El libro revisado "Mathematical Modelling of Zombies" es un libro divulgativo, que presenta modelos matemáticos aplicados a la epidemiologia por medio de analogías con las películas y novelas de terror de la ficción. Consta de 523 páginas repartidas en 17 capítulos, en el capítulo de introducción el editor se pregunta ¿Qué pueden enseñarnos los zombis acerca de matemáticas? Donde además de mencionar los diferentes modelos matemáticos que tienen aplicación en el modelamiento de enfermedades, nos comenta que su motivación para escribir el libro fue la gran recepción de un artículo que publicó en el 2009 con este mismo título. En cuanto al resto de capítulos y sus respectivos autores son:

- La propagación viral de una noticia zombi (Robert Smith?). Donde se utiliza el modelo SIR y las estadísticas de Google Trends para analizar la difusión de una noticia a través de los medios de comunicación.

- Los no muertos: ¿una plaga para la humanidad o una poderosa herramienta nueva para investigación epidemiológica? (Jane M. Heffernan y Derek J. Wilson). Realizan una analogía entre los zombis y vampiros respecto a enfermedades epidémicas y endémicas.

- ¡Cuando los zombis atacan! Final alternativo (Phil Munz). Presenta una variación del modelo depredador- presa de Lotka-Volterra para incluir tendencias cíclicas y fluctuaciones en las diversas poblaciones,

- ¡Cuando los humanos contraatacan! Estrategias adaptativas para los ataques zombi (Bard Ermentrout y Kyle Ermentrout). Describe un modelo biestable y con comportamiento oscilatorio que ocurre al suponer una ventaja de los humanos cuando la población zombi es baja (y viceversa), lo cual cambia cuando los humanos se vuelven complacientes al enfrentar pocos ataques.

- Aumentar la supervivencia en una epi- demia zombi (Ben Tippet). Construye un modelo epidemiológico en el cual analiza cual es la mejor estrategia a seguir ¿Es mejor correr y esconderse, enfrentamiento directo o seguir con la vida cotidiana?

- ¿Cuánto tiempo podemos sobrevivir? (Thomas E. Woolley, Ruth E. Baker, Eamonn A. Gaffney y Philip K. Maini). Usa un modelo de difusión con una población distribuida aleatoriamente en una región unidimensional para determinar los tiempos de interacción que producen una onda infecciosa.

- Demografía de zombis en EEUU (Daniel Zelterman). Analiza variables demográficas y las relaciona con las búsquedas en Google de un tema determinado (en este caso zombis).

- ¿No es seguro salir aun? Inferencia estadística en un modelo de brote zombi (Ben Calderhead, Mark Girolami y Desmond J. Higham). Presenta conceptos básicos de las técnicas estadísticas para calcular parámetros que no se pueden medir directamente.

- El zombi social: Modelando los brotes de no muertos en redes sociales (Laurent Hebért-Dufresne, Vincent Marceau, PierreAndré Noël, Antoine Allard y Louis J. Dubé). Considera las redes sociales y la adaptabilidad de las interacciones sociales.

- Sistema de alerta de infección zombi basado en un modelo de toma de desiciones de lógica difusa (Micael S. Couceiro, Carlos M. Figueiredo, J. Miguel A. Luz y Michael J. Delorme). Busca predecir ataques inminentes.

- ¿Hay un zombi maniaco cerca de usted? Mejor asuma que si (Nick Beeton, Alex Hoare y Brody Walker). Utiliza un modelo basado en agentes para describir la dinámica de la transmisión.

- Zombis en la ciudad: un modelo en el lenguaje de programación Netlogo (Jennifer Badham y Judy-anne Osborn). Considera los tres posibles resultados: zombis ganan, humanos ganan y punto muerto, mostran- 
do como los modelos basados en agentes complementan los enfoques analíticos.

- Una representación lineal evolutiva para simular las políticas del gobierno en los brotes zombi (Daniel Ashlock, Joseph Alexander Brown y Clinton Innes). Analiza los efectos de las acciones del gobierno, la población y la efectividad de una vacuna.

- Dinámica de posesión en la leyenda del buscador (Gergely Röst). Presenta un modelo de posesión en la que el muerto debe atacar un vivo cada día para no desaparecer y evalúa dos estrategias de respuesta.

- El enjambre zombi: epidemias en presencia de atracción y repulsión social (Evelyn Sander y Chad M. Topaz). Incorpora interacciones sociales que se presentan en organismos biológicos que se agrupan en enjambres.

El editor del libro Robert Smith? (el signo de interrogación es parte del nombre), es un matemático australiano, con doble nacionalidad incluyendo la canadiense que tiene un fuerte interés en temas biológicos y sociales como: Drogas antirretrovirales, vacunas, educación, medios de comunicación, enfermedades tropicales desatendidas, plantas, microbicidas, fermentación autociclo, fundamentos de la epidemiología teórica. Trabaja en el departamento de matemáticas de la Universidad de Ottawa y ha publicado libros de ficción relacionados con Doctor Who y un libro de texto Ilamado Modelling Disease Ecology with Mathemathics, el cual incluye una introducción amigable a las ecuaciones diferenciales impulsivas y un estudio de caso actualizado sobre zombis, junto con modelado en Matlab.

A pesar de la gran cantidad de enfermedades y condiciones particulares de las sociedades que las sufren, los modelos matemáticos utilizados resultan ser bastante generales y se aplican para diversos tipos de estas, como por ejemplo el VIH y la malaria. El primer paso para desarrollar un modelo consiste en comprender la biología, y lu- ego llegar a una conclusión por medio del análisis matemático.

Los zombis son muy cercanos a varias técnicas matemáticas, ellos forman enjambres, se mueven pseudoaleatoriamente; al otro lado de la balanza para combatirlos se requiere estimar parámetros, tomar decisiones con base a información imperfecta, y desarrollar políticas que evolucionen conforme cambie la situación (sistemas dinámicos). La formación de enjambres puede modelarse por ecuaciones integro-diferenciales, que ayudan a comprender como las personas o los zombis se agrupan y que efectos ocurren sobre la propagación de la enfermedad. El movimiento pseudoaleatorio (de un zombi tambaleante o de un virus aéreo) puede describirse por difusión, lo que requiere ecuaciones diferenciales parciales.

Zombis con necesidades específicas (comer cerebros), utilizan modelamiento basado en individuos, con el cual se puede simular numéricamente los efectos de muchos individuos en un ambiente urbano. Las interacciones entre zombis y personas se describen por medio de redes, que también dan cuenta de la propagación de una enfermedad en un barrio o por viajes internacionales. Las técnicas empleadas para modelar el efecto de la enfermedad sobre la población son de diversos tipos: pueden ser analíticas, numéricas o una combinación de ambas; y nos permiten enfrentar lo desconocido. Una pandemia puede ser terrible, pero conociendo como las vacunas detienen su avance y la estructura de la población. Las matemáticas nos ayudan a determinar cuáles esfuerzos de control serán más útiles y cuando aplicarlos, salvando vidas y generando un ahorro económico valioso en tiempos de emergencia.

El segundo capítulo del libro tiene como autores a Jane M. Heffernan y Derek J. Wilson, y trata como tema central el modelo SIR. Jane M. Heffernan es una matemática canadiense, profesora del departamento de matemáticas y estadística de la Universidad de York, directora del laboratorio 
de modelamiento de infecciones e inmunidad; y Derek J. Wilson es un bioquímico, profesor del departamento de química de la Universidad de York, director de un laboratorio de espectroscopia de masa que investiga estructura, función y dinámica de las proteínas.

\section{SIR - modelo para epidemias}

En el surgimiento de una enfermedad, cuando no está distribuida en toda la población, esta se puede separar en tres grupos:

- Susceptible (S) son personas sanas que pueden contraer la enfermedad. Al comienzo de un brote, la gran mayoría de la población estará en esta clase.

- Infectados (I) son miembros de la población que han contraído la enfermedad y que pueden transmitirla. En el pico de una epidemia, una fracción significativa de la población estará en esta clase.

- Retirados (R) son individuos que han tenido la enfermedad y sobrevivieron. Para la mayoría de las enfermedades epidémicas, como la peste bubónica, el cólera y los zombis, la clase retirada es una pequeña proporción de la población.

El movimiento entre las clases se rige por las siguientes tasas:

1. La infección mueve a los individuos entre las clases 'Susceptible' e 'Infectado'. La transmisión ocurre cuando una $\mathrm{S}$ y una I se encuentran, con una tasa $\beta$.

2. La recuperación mueve a los individuos entre las clases 'Infectados' y 'Retirados'. Esto ocurre con la tasa $\sigma$.

3. La pérdida de inmunidad mueve a los individuos de la clase 'Retirado' a 'Susceptible'. Esto ocurre con la tasa $\omega$.

4. Nacimiento / Muerte. Cada clase tiene sus propias tasas de natalidad y mortalidad, lo que resulta en la adición o eliminación de personas de cualquier clase. La muerte ocurre con tasas $d S, d l, d R$ y $\mu$ (muerte debida específicamente a la enfermedad); los nacimientos llegan con tasa $\lambda$. Por simplicidad, se supone que todos nacen susceptibles.

Resumiendo, el modelo esta dado por el sistema de ecuaciones diferenciales:

$$
\begin{aligned}
& \frac{d S}{d t}=\lambda-\beta S I-d_{S} S+\omega R \\
& \frac{d I}{d t}=\beta S I-d_{I} I-\mu I-\sigma I \\
& \frac{d R}{d t}=\sigma I-d_{R} R-\omega R
\end{aligned}
$$

Estas ecuaciones, pueden resolverse fácilmente utilizando GNU Octave, por medio del siguiente código:

$$
\begin{aligned}
& \text { function } x \text { dot }=\text { sir_eqn }(x, t) \\
& \text { \% Parameter values } \\
& \text { beta=0.1; } \\
& \text { mu=0.05; } \\
& \text { sigma=0.03; } \\
& \text { omega }=0.0005 ; \\
& \text { dS=0; } \\
& \text { dR=0; } \\
& \text { dl=0; } \\
& \text { lambda }=0 ; \\
& \% \text { Define variables } \\
& \text { s = x(1); } \\
& \text { y = x(2); } \\
& r=x(3) ; \\
& \% \text { Define ODEs }
\end{aligned}
$$


$\mathrm{ds}=$ lambda-beta*s${ }^{*} \mathrm{y}-\mathrm{dS}{ }^{*} \mathrm{~s}+$ omega* ;

$d y=$ beta*s*y-mu*y-dl*y-sigma*y;

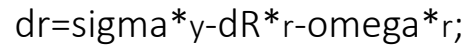

$\%$ Return gradients

$x$ dot $=[d s, d y, d r]$;

endfunction

$\mathrm{t}=$ linspace $(0,400,2001)+.1$;

$\mathrm{x} 0=[0.99,0.01,0]$;

$\mathrm{x}=$ Isode("sir_eqn",x0, t);

out=[transpose $(\mathrm{t}), \mathrm{x}]$;

$\operatorname{plot}(t, x(:, 1), "-r ", t, x(:, 2), "-g ", t, x(:, 3),,-b ")$

$x \lim ([0$ 400])

xlabel("Time","fontweight","bold")

ylabel("Number","fontweight","bold")

h = legend("S","I","R");

legend(h,"show")

\section{Conclusiones}

Este libro es una buena recomendación para aficionados a las películas o novelas de terror que quieran comparar lo que se presenta en ellas con modelos científicos relacionados, también recomendado para los matemáticos, médicos e ingenieros que deseen motivarse para estudiar los modelos epidemiológicos a partir de una lectura entretenida. Sin embargo, para este segundo grupo, este libro sería solo una introducción que podría ser complementada con el libro: "Modelling Disease Ecology with Mathemathics" del mismo autor, Robert Smith?

\section{Referencias}

Smith, R. (2014). Mathematical Modelling of Zombies. Canada: University of Ottawa Press.

Smith R. (2020). Homepage of Robert Smith?. Recuperado de: https://mysite.science.uottawa.ca/rsmith43/

Wikipedia. (2020). Jane Heffernan Recuperado de: https://en.wikipedia.org/wiki/Jane_Heffernan

Universidad de York (2020). Jane Heffernan, Recuperado de: http://immune. math.yorku.ca/jmheffer/biblio/tg/ U?page $=1 \&$ sort=type\&order $=$ desc

Derek J. W. The boss man. https://derekwilsonlab. $\mathrm{ca} /$ the-boss-man/

Epirecipes (2020). SIR model using Octave and LSODE. Recuperado de: http://epirecip.es/ epicookbook/chapters/sir/octave

Linge S., Langtangen $\mathrm{H}$. (2016). Spreading of diseases. Recuperado de: http://hplgit.github. io/Programming-for-Computations/pub/ p4c/._p4c-bootstrap-Matlab020.html

GNU Octave. Recuperado de: https://www.gnu. org/software/octave/download.html 